\title{
Use of imaging techniques for melanocytic naevi and basal cell carcinoma in integrative analysis (Review)
}

\author{
IOANA-ALINA GRAJDEANU ${ }^{1}$, DAN VATA ${ }^{1,2}$, LAURA STATESCU ${ }^{1,2}$, IOANA ADRIANA POPESCU ${ }^{1,2}$, \\ ELENA PORUMB-ANDRESE ${ }^{1,2}$, ADRIANA IONELA PATRASCU ${ }^{2}$, ALINA STINCANU $^{2}$, \\ TATIANA TARANU ${ }^{3,4}$, MARIA CRISAN $^{5}$ and LAURA GHEUCA SOLOVASTRU ${ }^{1,2}$
}

\author{
${ }^{1}$ Department of Dermatology, 'Grigore T. Popa' University of Medicine and Pharmacy, Faculty of Medicine, 700115 Iasi; \\ ${ }^{2}$ Clinic of Dermatology, Department of Dermatology, 'St. Spiridon' County Emergency Clinical Hospital, 700111 Iasi; \\ ${ }^{3}$ Department of Dermatology, 'Grigore T. Popa' University of Medicine and Pharmacy, Faculty of Dental Medicine, \\ 700115 Iasi; ${ }^{4}$ Clinic of Dermatology, Department of Dermatology, CF Iasi Hospital, 700506 Iasi; \\ ${ }^{5}$ Department of Dermatology, 'Iuliu Hatieganu' University of Medicine and Pharmacy, \\ 400000 Cluj-Napoca, Romania
}

Received January 27, 2020; Accepted February 27, 2020

DOI: $10.3892 /$ etm. 2020.8620

\begin{abstract}
Early detection of skin cancer is essential in order to obtain an improved prognosis. Clinicians need more objective and non-invasive examination methods to support their decision whether to biopsy or not tumoral lesions. These may include several imaging techniques such as dermoscopy, videodermoscopy, also known as sequential digital dermoscopy (SDD), computer-aided diagnosis (CAD), total body photography, imaging and high-frequency ultrasonography (HFUS), reflectance confocal microscopy, multiphoton tomography, electrical impedance spectroscopy, Raman spectroscopy, stepwise two-photon-laser spectroscopy and quantitative dynamic infrared. This review summarizes the current developments in the field of melanocytic lesions, such as naevi and basal cell carcinoma (BCC) imaging techniques. The aim was to collect and analyze data concerning types, indications, advantages and disadvantages of modern imaging techniques for in vivo skin tumor diagnosis. Two main methods were focused on, namely videodermoscopy and HFUS, which can be included in daily dermatologists' practice. In skin tumors HFUS allows the assessment of tumoral lesions with depth smaller than $1.5 \mathrm{~cm}$, being described a correlation between ultrasonographic depth and the histologic index.
\end{abstract}

Correspondence to: Dr Dan Vata, Department of Dermatology, 'Grigore T. Popa' University of Medicine and Pharmacy, Faculty of Medicine, Str. Universitatii 16, 700115 Iasi, Romania

E-mail: danvata@yahoo.com

Key words: skin tumors, imaging techniques, videodermoscopy, high frequency ultrasonography, integrative analysis

\section{Contents}

1. Introduction

2. Videodermoscopy and computer-aided diagnosis (CAD) in melanocytic naevi and basal cell carcinomas (BCCs)

3. High frequency ultrasonography of cutaneous melanocytic naevi and basal cell carcinomas

4. Other techniques

5. Conclusions

\section{Introduction}

Imaging techniques are being increasingly used in the non-invasive evaluation of various cutaneous diseases. They possess a complementary role in the in vivo assessment of cutaneous lesions and can support clinicians in their diagnosis and therapeutic decisions. There are several imaging methods apart from classical photography including manual dermoscopy, videodermoscopy or sequential digital dermoscopy (SDD), computer-aided diagnosis (CAD), total body photography, high-frequency ultrasonography (HFUS), reflectance confocal microscopy, multiphoton tomography, electrical impedance spectroscopy, Raman spectroscopy and quantitative dynamic infrared imaging (1). They can be classified as follows: Tools useful in skin tumor screening in daily clinical routine, tools for the examination of preselected lesions in order to obtain an automated diagnostic score, tools for the examination of preselected lesions at specialized centers and devices at an experimental stage of development (2). The aim of this review was to collect and analyze data concerning non-invasive diagnostic strategies for cutaneous tumors, such as naevi and basal cell carcinoma (BCC) diagnosis, focusing on two main methods, namely videodermoscopy and HFUS. This study was approved by the Clinical Research Ethics Committee of 'St. Spiridon' County Emergency Clinical Hospital (Iasi, Romania) and by the Research Ethics Committee of 'Grigore T. Popa' University of Medicine and Pharmacy (Iasi, Romania). 
Written informed consent was obtained from all patients prior to publication.

Videodermoscopy and HFUS can be used in daily clinical dermatologist practice in order to reduce invasive procedures, like biopsies and fine needle aspirations $(2,3)$. Videodermoscopy using computer technology may be used to store images, indirectly evaluate and extract features in order to distinguish melanoma from other pigmented benign skin lesions. HFUS offers in vivo preoperative details and represents a useful method to investigate superficial macular and nodular cutaneous lesions with depth lower than $1.5 \mathrm{~cm}$. One benefit is that it helps in the evaluation of lesions when dimensions are too small to be assessed by other imaging methods, such as computed tomography or magnetic resonance imaging $(2,3)$.

\section{Videodermoscopy and CAD in naevi and BCC}

The term 'dermoscopy' (synonym: epiluminescence microscopy) refers to surface microscopy and currently indicates all methods that offer visualization at 10-fold magnification of skin tumoral lesions using optical magnification and fluid immersion or polarized lighting. Videodermoscopy represents digital dermoscopy and this method allows dermoscopic visualization of tumoral structures in higher magnification. Dermoscopy improves the ability of physicians to differentiate the various types of melanocytic naevi from one another and to diagnose melanoma. The main principle of dermoscopy screening is the examination of all cutaneous lesions, not only pigmentary or nonpigmentary lesions chosen by the patient or preselected for clinical examination.

Argenziano et al (4) proposed a new dermoscopic classification of naevi into seven groups:

i) Globular/congenital naevi with globular pattern in children reveal brown globules inside the lesion and especially at the periphery. It may sometimes also show hypopigmentation or a structureless brown pigmentation. In adults, these types of naevi appear elevated, with a papillomatous surface and dermoscopically present a cobblestone pattern or sometimes a fried-egg pattern characterized by a regular pigment network with globules, cobblestone or structureless area in the center and a flat portion at the periphery.

ii) Reticular naevi are late-acquired melanocytic naevi and can be considered small $(<6 \mathrm{~mm})$ or large $(\geq 6 \mathrm{~mm})$, having a flat or elevated surface with a brown to black color. Dermoscopically, they present a regular pigment network with or without areas of hypopigmentation and/or structureless brown to black coloration. Large lesions may have multiple hypo- and/or hyperpigmented areas.

iii) Pigmented Spitz or Reed naevi are brown-black, flat to elevated, symmetrical lesions which are characterized by a star-burst pattern seen by dermoscopy with radiating pattern appearance (multiple streaks of pigmentation and symmetrical large globules at the periphery). In no pigmented lesions are dotted vessels and reticular depigmentation seen.

iv) Blue naevi by naked eye examination are blue to black papules, nodules with variable size and dermoscopy reveals a homogeneous pattern of structureless blue coloration.

v) Site-related naevi: a) Acral naevus: Brown to black, flat to slightly elevated tumoral lesion on palms/soles showing in parallel, parallel-furrow, lattice-like or fibrillar pattern. b) Facial naevus in children may appear as a flat or elevated, brown symmetrical lesion, smaller than $15 \mathrm{~mm}$ with a dermoscopic pseudoreticular pattern. In adults, it may appear as an elevated and skin colored lesion with the presence of comma vessels in dermoscopy.

vi) Naevi with special features: a) Irritated naevus shows a reticular, globular or structureless pattern with grey and red pigmented areas. b) Naevus with eczematous halo may present reticular, globular or structureless pattern with yellow areas and eczematous changes within a naevus. c) Combined naevi show at least two of the following patterns: reticular, globular, homogeneous or starburst. d) Recurrent naevus with atypical pigmentation pattern and scar-like structures. e) Halo naevus has a globular pattern with blue pepper-like granules and/or white scar-like areas.

vii) Unclassifiable melanocytic lesions: one of the previous type of naevi which shows atypical features (in this cases a melanoma cannot be excluded) (4).

Melanocytic naevi represent direct precursor lesions for melanoma and 20-30\% of melanomas develop on a nevus (5). A high total nevus count is a risk for melanoma and ongoing surveillance of naevi for malignant degeneration is needed (6). Melanoma is a high-risk skin cancer and it has potential to metastasize, while basal cell carcinoma has the potential to infiltrate the surrounding tissue. Melanoma screening strategies have the objective of a total body examination and Breitbart et al (7) reported that improving melanoma prognosis is based on early detection. Two meta-analyses have demonstrated that dermoscopy can improve the sensitivity for recognition of cutaneous melanomas in comparison to examination with the naked eye $(7,8)$. Videodermoscopy increases the effectiveness of melanoma screening methods and consists of a modern method of capturing and sequential monitoring of digital dermoscopic images of single or multiple cutaneous lesions. The initial images printed or stored electronically are used in comparison with future examinations. The utility of videodermoscopy for monitoring pigmented skin lesions was for the first time reported by Stolz et al (9) and Braun et al (10). Haenssle et al (11) claimed that SDD was especially useful in the evaluation of high risk patients and this investigation improved the sensitivity and positive predictive value of melanoma screening at follow-up examinations in comparison with dermoscopy alone. Multiple studies reported values of sensitivity examination between 83 and $84 \%$ (10). Other benefits of SDD consist in identifying initial featureless melanomas, monitoring the naevi and decreasing the number of unnecessary biopsies. Indirect advantages represent improving the patient-dermatologist relationship and may help also in improving follow-up visit compliance $(12,13)$. However, there are limitations of SDD, such as increasing the time of examination, costs, requiring expert examiners, the possibility of occurrence of taking image problems and equally possibility of monitoring melanomas instead of biopsies. Dermoscopic algorithms including the ABCD rule, pattern analysis or the 7-point checklist may not reliably differentiate in situ melanomas from melanocytic naevi (14). Similarly, there is a lack of consensus regarding which lesions should be analyzed $(15,16)$. Kittler et al (17) recommend that short-term follow-up (3 months) should be indicated in suspicious lesions that do not present features of melanoma and biopsy is recommended when lesions morphology changes. The selection of pigmented 
skin lesions for SDD follows clinical and classical dermoscopic examination of all cutaneous lesions. Lesions with macroscopic signs of atypia (asymmetric form, inhomogeneous color, irregular borders) and/or dermatoscopic signs of atypia, such as prominent pigmentary network, focal eccentric hyper/hypopigmentation or multiple components have indication for short follow-up. Poorly accessible locations may also have indication for follow-up. Long-term follow-up (6-12 months) should be indicated for patients with multiple atypical naevi or high-risk individuals (17). Risk factors for the development of melanoma mentioned in epidemiologic studies include a positive patient or family history of melanoma, the number of typical and atypical naevi, the Fitzpatrick skin, intermittent UV exposure and the presence of other non-melanoma skin cancer (18).

In a study of pigmented lesions, which had dynamic alterations in follow-up, the reported ratio of excised cutaneous melanomas to benign naevi was 1:79 in the group of patients with multiple typical naevi, while in the group with multiple atypical naevi, the ratio was 1:15. Similarly, melanomas could be diagnosed earlier using SDD rather than clinical examination and conventional dermoscopy (mean registered Breslow depth was 0.41 vs. $0.62 \mathrm{~mm} ; \mathrm{P}=0.04)$ (19). Therefore, periodic control examinations using SDD are useful particularly in evaluating patients with multiple atypical naevi. Studies in cases of patients without an increased risk of melanoma did not demonstrate advantages from skin pigmented lesions dermoscopic follow-up because many benign lesions were excised and no melanomas were diagnosed (20).

Dermoscopically false positive and false negative tumors exist and false positive diagnosis may lead to unnecessary excisions. Otherwise false negative diagnosis is much more dangerous, since it might fail to notice a cancer, with serious consequences for both the patient and the dermatologist. On the one hand, irritated or regressive seborrheic keratosis, melanoacanthoma, solar lentigo, thrombosed angioma, dermatofibroma, benign adnexal tumors and certain type of naevi such as Clark, Spitz, recurrent, combined, blue or sclerosing are the most frequent benign tumors that may display dermatoscopic characteristics suggestive of malignancy. Dermoscopy and, similarly, SDD may reveal certain features for benignity: solar lentigo has a broad network; Clark nevus has a clinical context; Spitz naevi are characteristic for young age, recurrent naevi present pigmentation within the scar, combined naevi may show a bluish central area, blue naevi have usually a certain onset and sclerosing naevi most frequent have location on the upper back. On the other hand, malignant tumors that might mimic benign tumors are represented by melanoma with the following clinical types: In situ, nevoid, amelanotic, spitzoid, regressive or verrucous; squamous cell carcinoma and basal cell carcinoma with non-pigmented clinical forms. Dermoscopy and SDD may give the following clues to recognize the melanoma subtypes: Irregular hyperpigmented areas appear in melanoma in situ, history of growth is characteristic for nevoid melanoma, pink color and irregular linear vessels accompanied by dotted vessels are suggestive for amelanotic melanoma, blue-black sign appears in verrucous melanoma and peppering or scar-like depigmentation may reveal a regressive melanoma (21) (Fig. 1).

CAD represents artificial intelligence-based techniques that are using a computer to analyze images and to determine

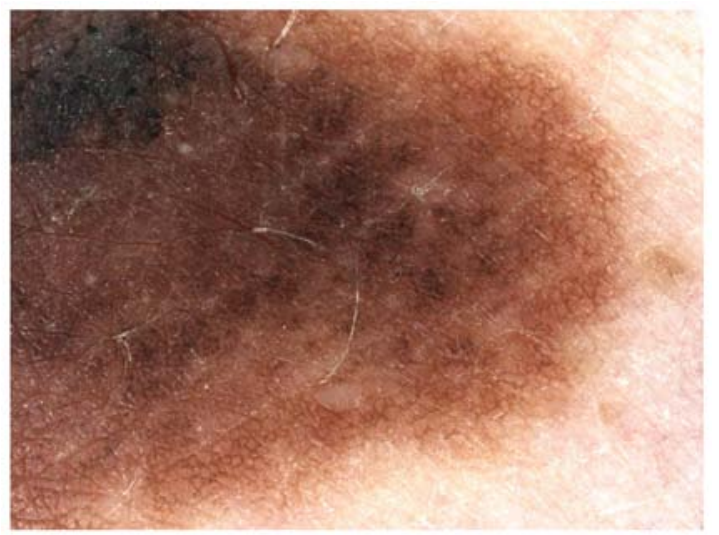

Figure 1. Videodermoscopy image of an atypical nevus in a patient with multiple atypical naevi syndrome: Signs of atypia such as prominent pigment network, focal eccentric hyperpigmentation (magnification, x15).

the risk of malignancy. In primary care, CAD may help general practitioners to identify high-risk lesions and may reduce unnecessary excisions without missing melanoma cases. Computer analysis of single or multiple pigmented skin lesions includes preprocessing images with artefacts (hair, air bubbles, specular reflections), image enhancement, respectively, color calibration and illumination correction. The other steps include lesions border detection, comparison of segmentation algorithms, feature extraction, registration and change detection, lesions classification, use of CAD systems with digital dermoscopy analysis instruments and 3D lesions analysis (22).

A review of 42 studies evaluating digital dermoscopy-based CAD systems (Derm-CAD) in 9,602 lesions (with detection of 1,220 melanomas, 83 basal cell carcinomas and 9 squamous cellular carcinomas) evaluated the accuracy of CAD systems for diagnosing cutaneous invasive melanoma and atypical intraepidermal melanocytic naevi (20). The results showed that in highly selected patient populations all CAD types demonstrate high sensitivity. CAD systems were useful as a back-up for specialist diagnosis to assist in minimizing the risk of missing melanomas. Nonetheless, the evidence base was too poor to understand whether CAD system outputs translate to different clinical decision-making in practice (23).

SDD can be improved by telediagnosis, which may provide a better examination of pigmented cutaneous tumoral lesions carried out by primary care physicians, not expert in that kind of diagnosis, thus reducing the number of consultations in specialized centers. Telediagnosis is usually used in highly specialized centers, while in smaller and non-specialized units surgical treatment is usually performed in cases of pigmented lesions suspicious for malignancy (24).

$\mathrm{BCC}$ is the most common type of skin cancer in the world and can be associated with significant morbidity, especially if left untreated. BCC can present a variety of clinical morphologies, such as erythematous patches to ulcerated nodules. Depending on the subtype of $\mathrm{BCC}$ and the degree of pigmentation, the clinical differential diagnosis can range from benign inflammatory conditions to melanoma and dermoscopy has dramatically improved the clinicians' diagnostic accuracy for both clinical pigmented or non-pigmented tumoral types. There are multiple histopathologic subtypes of BCC including 
superficial, nodular, morpheaform or sclerosing or infiltrative BCC. Fibroepithelioma of Pinkus, microcystic adnexal and basosquamous cell BCC are similarly defined as histopathologic subtypes. The main dermoscopic criteria of BCC are represented by classical arborizing vessels; fine or short arborizing vessels, focused dots, large blue gray ovoid nests, multiple blue gray globules, concentric structures, leaf-like areas, spoke-wheel areas, small erosions or ulceration, pink-white areas and short white streaks. The evaluation of vascular pattern may facilitate discrimination between basal cell carcinomas subtypes and it is reported that aggressive tumoral forms have less or no pink coloration and a lack of central vessels (25). Digital dermoscopy of superficial basal cell carcinoma usually reveals short, fine 'microarborizing' telangiectasia, multiple small erosions, shiny white-to-red, translucent, opaque structureless areas and brown-colored pigmented structures. Nodular basal cell carcinoma displays large arborizing vessels, large ulcerations and blue-gray ovoid nests in pigmentary forms. While vessels of BCC are bright red and arborizing, vessels outside the tumors belonging to the normal dermal plexus have a blurred aspect and a darker hue. Sclerodermiform BCC presents branching vessels, which are usually finer, more scattered and show fewer branches compared to the classic vessels of nodular BCC. Moreover, the underlying fibrosis induces a whitish background, whereas nodular BCC typically reveals a translucent pinkish color. The differentiation between tumoral vessels and the vascular pattern of normal skin is needed in order to estimate the lateral extension of BCCs, which may have clinically ill-defined borders (26-28).

SDD is similarly used for monitoring superficial CBC response to very popular non-ablative treatments. Clinical evaluation after therapy may not be reliable, but dermoscopy offers information on the possible residual tumoral lesions (29). The disappearance of the BCC dermatoscopic criteria has shown histopathologic clearance, while the presence of the same or new BCC dermoscopic criteria correlates with persistence or tumor reoccurring. Criteria such as arborizing vessels, ulceration or blue-gray ovoid nests and maple leaf-like areas with pigmented structures may predict residual disease. Red or white structureless areas and superficial fine telangiectasia correspond to equivocal features (30). The detection of blue-gray globules has been reported to indicate early recurrence of $\mathrm{CBC}$ (23). In a study with a series of BCCs treated with imiquimod, criteria like arborizing vessels, spoke-wheel areas, maple leaf-like areas were reported to also decrease in size and number after treatment initiation, whereas structures such as multiple blue-gray globules and ovoid nests were detected for a longer period of time (31).

\section{High frequency ultrasonography of melanocytic naevi and basal cell carcinoma}

Ultrasonography has been used in dermatology for nearly 40 years. Alexander and Miller introduced ultrasonography as a non-invasive technique to appreciate normal skin thickness and in 1980 it was used to assess skin nodules and cutaneous diseases (32). This procedure is a method allowing the in vivo histologic evaluation of the cutaneous structure. It is based on the phenomenon of transonic wave reflection in the form of an imaging gray scale for interpretation, in accordance with the skin characteristics represented by the percentage of collagen, keratin and water in tissues. The indications of this imaging technique are multiple and they include the evaluation of benign or malignant tumors, melanocytic lesions, as well as inflammatory diseases. Important data regarding size, structure, elasticity and vascular flow of cutaneous lesions can be obtained by using conventional 2D ultrasound, HFUS, Doppler ultrasound, contrast enhanced ultrasound and elastography. Elastography represents a non-invasive technique that offers information on the soft tissue elasticity and a reduced elasticity is correspondent to hypervascularization and tumor congestion $(32,33)$. Tumor macrocirculation can be evaluated using color Doppler flow map for vessel enhancement and pulse Doppler is indicated in differentiating between vein and arteries in study of velocity (3).

HFUS using 20-100 MHz transducers constitutes a modern procedure that is used for skin investigation. Studies show that HFUS is superior to clinical examination alone, since it provides information in measurement of size and appreciation of contour, structure and assessment of skin lesion depth (31). The high frequency transducer offers an 80-micrometer axial resolution and a 200-micrometer lateral resolution. Using transducers with $20 \mathrm{MHz}$, epidermis is a hyperechogenic entry line, which varies according to age, anatomical area or the topical therapy. The dermis is markedly echogenic and sharply demarcated from the hypoderm, which is hypoechoic. Adipose panniculi are separated by echogenic conjunctive vascular septae. Skin tumor HFUS allows the assessment of macular and nodular lesions with depth smaller than $1.5 \mathrm{~cm}$. Epidermis, dermis, hypodermis, dermoepidermic and dermohypodermic junctions are identified and allow correlation of the ultrasonographic depth and the histologic index. This correlation is limited in presence of perilesional inflammatory infiltrate $(1,34,35)$. Pigmentary tumoral lesions, such as naevi, melanomas and nonpigmentary tumoral lesions, including carcinomas, can be assessed and described in detail using ultrasound. Most of the skin tumoral lesions appear as hypoechoic cutaneous or as subcutaneous thickening on HFUS. Melanocytic naevi are hypoechoic, symmetrical and usually well delimited from the adjacent dermis; they may present many small echoes. Junctional naevi are very thin, whereas dermal naevi are thicker. In congenital pigmentary naevi, ultrasonography is a useful tool in monitoring and early detection of possible malignant transformation. Acoustic shadowing and retrolesional echogenicity is suggestive for melanoma (36). Similarly, cutaneous ultrasound can support the differential diagnosis between blue naevi and metastases of melanoma. Blue naevi appear hypoechoic, homogeneous, 'dish-shaped' lesions and are located in the superficial dermis, whereas melanoma metastases are hypoechoic, heterogeneous lesions, 'potato-shaped' located in the hypodermis (37). Malignant melanoma has a high mortality rate and the histological depth of tumor or Breslow index represent the most important factor for prognosis and therapy. Thin malignant melanoma is represented by irregular hypoechogenic aspect band and HFUS can reveal the degree of dermic penetration with possible visible areas of vertical growth towards dermis and inflammatory infiltrate with hypoechogenic aspects. Similarly, visible areas of tumoral regression accompanied by 
fibrosis of hyperehogenic aspect can be identified (33). Color and Power Doppler studies may help to evaluate vascularity of lesions. Nodular malignant melanoma is described as a hypoechogenic nodular tumor and HFUS quantifies the degree of the dermis invasion. Regarding therapeutic management and prognosis, melanomas with depth index smaller than $1 \mathrm{~mm}$ defined as thin melanomas have a good prognosis, with a 95 to $100 \%$ chance of a 5-year-survival after surgical excision with a margin of $1 \mathrm{~cm}$. Ultrasonography is useful in melanoma patients staging ( $\mathrm{T}$ stage) and both conventional and Doppler ultrasound are helpful in the quantification of $\mathrm{N}$ stage melanoma identifying possible positive adenopathy before performing sentinel lymph node biopsy.

According to literature, $\mathrm{BCC}$ is the most common skin cancer representing $75-90 \%$ of all skin cancers (35). Echography helps in the clinical diagnosis and shows hypoechoic masses, which replace the collagen (more hyperechoic) with tumor cells (lower density). It may estimate tumor size (depth and diameter), delimitation of presurgical margins and helps in surgical planning (study of peritumoral blood vessels). It may also provide information on invasion of adjacent structures (cartilage and/or bone) and similarly help to evaluate the response to non-surgical treatments and study of recurrences. High frequency $20 \mathrm{MHz}$ ultrasound shows an oval solid hypoechoic or anechoic tumor with irregular borders and hyperechoic points. Usually, BCC is well delimited from the surrounding dermis and dermis invasion can be quantified. Color Doppler shows moderate increase in intra and peritumoral vascularization. The hyperechoic spots may be useful in order to differentiate BCC from other types of skin cancer. On histologic analysis, the hyperechoic points appear to correlate with the presence of horn cysts, microcalcifications or clusters of apoptotic cells in the center of nests of basal cell carcinomas. Wortsman described ultrasound as a first-line imaging modality for the management of facial cutaneous BCC (38). Preoperative imaging in facial BCC may aid surgical therapeutic plan, especially in high risk areas of recurrence such as eyes, nose and ears, in case of incomplete excisions. Risk factors for incomplete excision are head location, multiple tumors, morpheiphorm and infiltrative subtypes (39-42).

A study conducted on 56 patients with BCC reported lower ultrasonographic index in comparison with histologic index, but a moderate correlation index was obtained and statistically significant differences were not identified (43). HFUS can be similarly useful in detecting subclinical satellite lesions. In another study with 46 subjects diagnosed with BCC (18 patients); superficial spreading melanoma (8 patients) and nodular melanoma (20 patients), the ultrasonographic depth index was comparable to the histological one, with a very good sensitivity (98-99\%) (43). BCC with depth index smaller than $1.5 \mathrm{~mm}$ may benefit from photodynamic therapy (35). HFUS provides real-time data on depth and lateral invasion and their examination show hypoechoic inhomogeneous tumors with possible ulceration (43). In addition, HFUS offers more accurate depth index in comparison to conventional ultrasonography $(39,44)$.

The benefits of using HFUS consist of repeatability, the lack of risk for patients, being a non-invasive method with minimal costs and also the intake of morphological details of cutaneous lesions that cannot be obtained from clinical or histological examination $(41,45)$. Depth, area and demarcation from the adjacent structures can be described with the possibility of identifying a preoperatory prognosis. It is also admitted that HFUS images could give important information on internal structure especially the collagen and keratin pattern. In a retrospective study in which the skin tumor evaluation protocol was completed with HFUS, the accuracy of clinical diagnosis was estimated to increase from 73 to $97 \%$ (46). The estimation of tumor margins is important in therapy planning and may avoid incomplete excision and surgical reintervention. In addition, imaging findings can be useful in the follow-up after cryotherapy or laser treatment (36) and it is an easily and well accepted method for follow-up of patients (36).

In contrast, ultrasonographic histologic differentiation of skin tumors, either benign or malignant, is not always possible according to literature data $(46,47)$. It has very good sensitivity, but low specificity (35). This technique requires modern devices and it is a time-consuming technique for the examiner. HFUS is operator sensitive and its accuracy depends on the examiner's level of training in ultrasonography. Besides, errors may appear if the pressure used during examination is not appropriate. Another limit is that in situ tumors situated only in epidermis, such as in situ melanoma and thin melanoma, may not be detectable and ultrasound examination usually does not differentiate between melanomas and clinically atypical naevi (34). In addition, the tumoral thickness is not accurate if tumoral structures have important perilesional inflammatory infiltrate. It has been reported that ultrasonographic values could be slightly overestimated due to inflammatory infiltrate associated to the tumor, as well as skin hypertrophied sebaceous glands or hair follicles $(48,49)$. Similarly, the identification of small infiltrative dermic tumoral lesions is another reported limitation of the technique (50) (Fig. 2).

Pellacani and Seidenari (49) used a combined approach based on sonography and clinical-videomicroscopy in order to evaluate preoperatively thick melanomas. Echographic thickness using $20 \mathrm{MHz}$ sonography was calculated for each tumoral lesion. Two clinical features (nonpalpability for thin melanomas and clinical regression for thick melanoma) and seven videomicroscopic features were identified for distinction between thick and thin melanomas. Central pigment network, central brown globules and blotches were considered features of thin melanomas, while localized peripheral pigment network, grayish polygonal areas, veil and vessels pattern were features of thick ones. In this algorithm, a coefficient was attributed to each variable, a score was obtained for each tumor and a validated test for preoperative thickness prediction was finally developed. This test enabled the distinction of thick melanomas with $86.7 \%$ sensitivity and $100 \%$ specificity (49).

\section{Other techniques}

Reflectance confocal microscopy is a novel non-invasive diagnostic technique based on focal point illumination and visualization of different skin layers with possible differentiation of benign skin lesions from malignant lesions, which is also used for inflammatory skin disease diagnosis (50-52).

Lentigo maligna is characterized by the disruption of the typical honeycomb or cobblestone pattern in epidermis, multiple large round pagetoid cells and large nucleated cells 

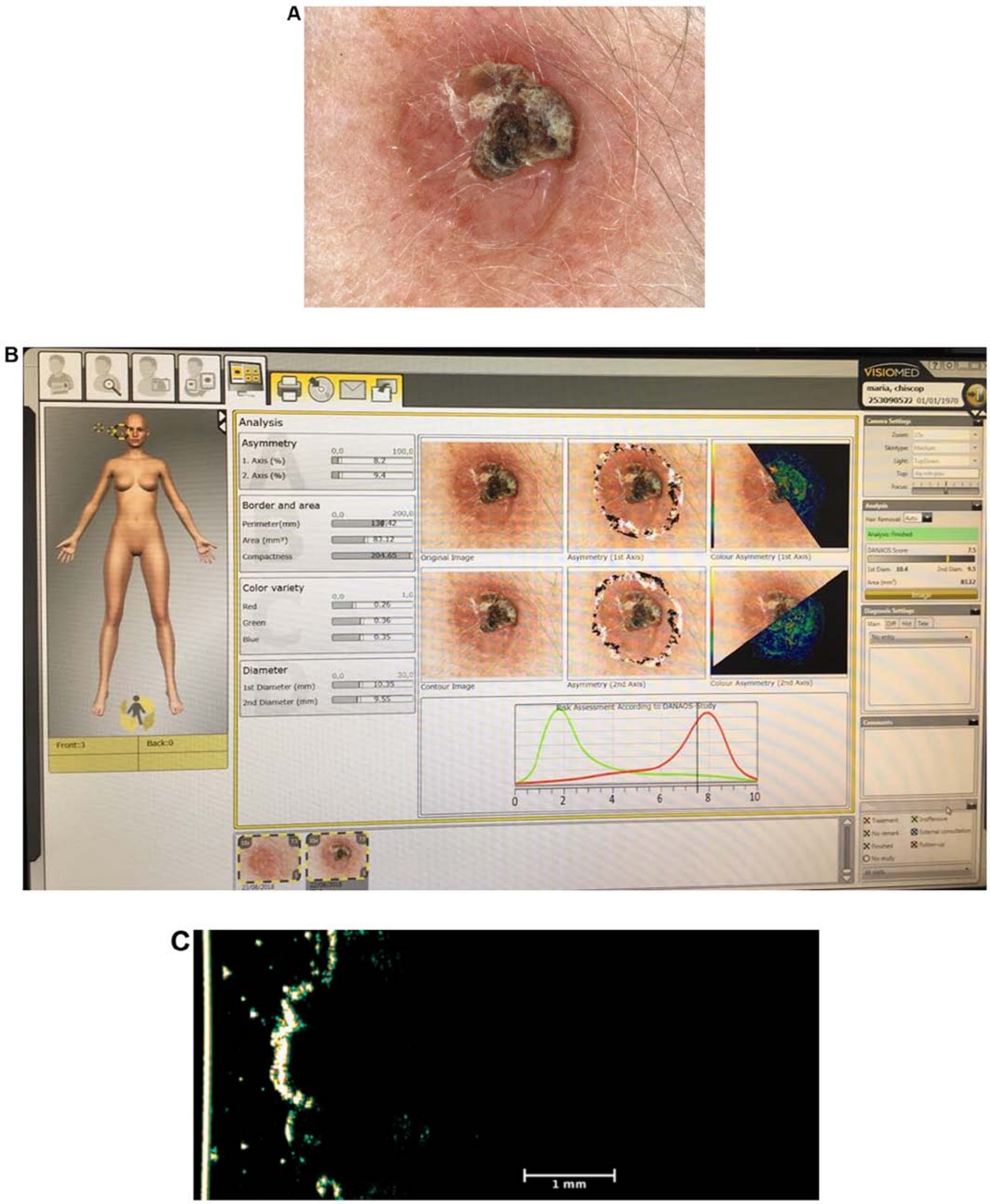

Figure 2. Imaging analysis of basal cell carcinoma. (A) Videodermoscopy image (magnification, x15): Positive features include presence of arborizing vessels, ulceration and hematic crusts. (B) Computer-aided analysis: Data on tumour diameter, colour and asymmetry. (C) HFUS analysis with Dermascan $\mathrm{C}^{\circledast} \mathrm{B}$ mode with $20 \mathrm{MHz}$ : Hypoechoic structure with vertical diameter of $10.88 \mathrm{~mm}$ and horizontal diameter of $5.169 \mathrm{~mm}$. HFUS, high-frequency ultrasonography.

in dermis $(53,54)$. In melanoma, the epidermis has irregular honeycomb or cobblestone patterns with large nucleated pagetoid cells. Similarly, dermoepidermal junctions are disorganized and the dermis has dense and sparse nests of cells, 
plump bright and small bright cells with increased reticulated fragmented bright collagen fibers. Pellacani and colleagues (55) analyzed 351 melanocytic lesions including melanoma and melanocytic naevi from 332 patients to obtain diagnostic accuracy. A sensitivity of $92 \%$ and specificity of $69 \%$ were reported (55). Spitz nevus represents a melanocytic lesion difficult to diagnose. In in vivo confocal microscopy, an atypical Spitz tumor is indicated by large conflating tumor nests with isolated epithelioid pleomorphic cells within the spinous layer, inhomogeneous nests, combined melanocytic tumor and melanocytic proliferation with elongated rete ridges (56).

In non-melanocytic tumors, such as BCC, confocal microscopic features include cells with elongated nuclei orientated at the same axis with separation of tumor islands from the surrounding stroma. Reflectance microscopy examination of cystic BCC may reveal solid tumoral masses appearing less bright than surrounding stroma, large dark areas with bright structures inside the center and periphery of tumoral lobules and numerous enlarged vessels. Similarly, in the inner portion of tumoral masses a peripheral palisade arrangement of elongated, polarized nuclei and bright round-oval structures, thin filaments and large dendritic cells may be observed (57). The progression of BCCs may be repeatedly monitored using this technique (58). Squamous cell carcinoma shows parakeratosis and hyperkeratosis in the epidermis, an atypical 'honeycomb' pattern with uneven dysplastic keratinocytes and detached keratinocytes (59). Dilated and tortuous vessels, perivascular inflammatory cells with shiny appearance may also be observed. The papillary dermis exhibits dark areas corresponding to blood vessels containing erythrocytes appearing as white central elements and also bright perivascular elements of inflammatory infiltrate may be noted (60). In Bowen disease, an acanthotic epidermis with large cells with bright center and dark peripheral halos may be observed. Similarly, there are remarked cells with dark center and bright rim surrounded by a dark hallo, which are related with dyskeratotic cells on histological examination (61).

The limitations of the reflectance confocal microscopy technique include the fact that diagnosed lesions are limited to the upper dermis up to 250-300 $\mu \mathrm{m}$ and is operator sensitive (53).

Multiphoton tomography is a tissue imaging method based on the tissue exposure with intense near infrared laser pulses resulting in two major signals (the autofluorescence and second harmonic generation). These signals are used to image cells and the extracellular matrix. Indications of the method include early detection of skin cancer and other tissue pathologies, obtaining non-invasive tissue histology within minutes. The use of multiphoton imaging methods for skin tumor diagnosis and monitoring shows considerable promise. Several studies $(62,63)$ have reported that tumors can be identified through a variety of different contrast mechanisms and may be used in the investigation of suspicious naevi or other neoplasms, such as melanoma, BCC or squamous cell carcinoma, especially in locations such as the head and neck, where surgeons need effective imaging tools to determine the whole lesion margin in order to resect it completely. The combination of multiphoton microscopy with other imaging modalities, such as ultrasound imaging, confocal microscopy and optical coherence tomography have been shown to be useful in skin research (63).
Electrical impedance spectroscopy is a noninvasive method that helps in diagnosing skin cancer using a handheld probe with an electrode applied directly on the skin. The basic principle is based on the utilization of electrical impedance variations to differentiate between normal skin and tumoral lesions. It has indication in lesions, which have clinical or dermoscopic suspicious features (64).

Raman spectroscopy represents a noninvasive tool used in vivo in skin cancer diagnosis that captures unique optical signals via molecular vibrations in tissue samples (65). Zhang et al (66) suggested in a meta-analysis that Raman spectroscopy could be an accurate tool for differentiating melanoma, BCC and squamous cell carcinoma from normal tissue. In a cohort with 645 confirmed lesions from 573 patients with skin tumors, pretumoral lesions and benign skin lesions were included. They were divided into a training cohort $(n=518)$ and testing cohort $(n=127)$ and it was found that the diagnostic tumor specificity used for fixed sensitivity was improved from $0.17-0.65$ to $0.20-0.75$ (67).

Other technologies (stepwise two-photon-laser spectroscopy, quantitative dynamic infrared imaging, in vivo multiphoton tomography, infrared thermal image-analysis, epidermal genetic information retrieval) are on the verge of becoming less experimental, yet more clinically applicable for diagnosing skin cancer.

\section{Conclusions}

The early accurate diagnosis of skin cancer is essential to guide the appropriate management and to improve the morbidity and survival rates. None of the presented imaging techniques is able to provide a certain and final diagnosis or to completely replace the histopathological examination. Up to date, the need for a complete skin cancer screening fully provided by automated devices has not been satisfied (2).

Videodermoscopy or SDD has distinct advantages over malignant tumor screening. This technique improves sensitivity and specificity of melanoma detection and represents a complementary examination method to improve early detection of melanomas particularly in patients at risk. Dermatologists should use a combination of history, clinical examination and SDD in order to be effective in the diagnosis of initial malignant tumor lesions. Digital imaging applications in both naevi and BCCs have real benefits: Objective non-invasive documentation of tumoral lesions, digital dermatological image archives, telediagnosis, quantitative description of clinical features of cutaneous lesions and 3-dimensional reconstruction. Dermoscopic malignant skin tumor diagnosis is limited in the diagnosis of very early and mainly featureless melanomas. Although automatic diagnosis systems are not perfect yet, their most valuable functionality has already been achieved in the capacity of description of lesion characteristics.

HFUS represents a non-invasive, reliable method that can be complementary utilized in the physical examination for the assessment, diagnosis and management of cutaneous tumors.

Although the gold standard method of diagnosing skin tumors is histopathological examination, non-invasive methods such as SDD and HFUS, allow a multimodal approach and offer the opportunity of presurgical tumor evaluation and the establishment of prognostic factors and therapeutic management (68). 


\section{Acknowledgements}

Professional editing, linguistic and technical assistance performed by Irina Radu, Individual Service Provider, certified translator in Medicine and Pharmacy (certificate credentials: series E no. 0048).

\section{Funding}

No funding was received.

\section{Availability of data and materials}

Imaging data were provided using MicroDermVisiomed ${ }^{\circledR}$ system analysis and Dermascan $\mathrm{C} \mathrm{USB}^{\circledR}(20 \mathrm{MHz}$ B-mode) equipments. The datasets used and/or analyzed during the current study are available from the corresponding author on reasonable request.

\section{Authors' contributions}

All authors contributed to the acquisition of the data and critical revision of manuscript for important intellectual content. AS conceived review on dermoscopy. IAG and LGS performed videodermoscopy, HFUS techniques and wrote the manuscript. LS, IAP, DV wrote and conceived review sections on dermoscopy and MC wrote and conceived review on ultrasound. EPA, AIP and TT searched data on the other imaging techniques. All authors read and approved the final version of the manuscript.

\section{Ethics approval and consent to participate}

This study was approved by the Clinical Research Ethics Committee of 'St. Spiridon' County Emergency Clinical Hospital (Iasi, Romania) and by the Research Ethics Committee of 'Grigore T. Popa' University of Medicine and Pharmacy (Iasi, Romania). Written informed consent was obtained from all patients prior to publication.

\section{Patient consent for publication}

Written informed consent was obtained from all patients prior to publication.

\section{Competing interests}

The authors declare that they have no competing interests.

\section{References}

1. Korotkov K and Garcia R: Computerized analysis of pigmented skin lesions: A review. Artif Intell Med 56: 69-90, 2012.

2. Fink $\mathrm{C}$ and Haenssle HA: Non-invasive tools for the diagnosis of cutaneous melanoma. Skin Res Technol 23: 261-271, 2017.

3. Crisan D, Badea AF, Crisan M, Rastian I and Solovastru Gheuca L: Integrative analysis of cutaneous skin tumours using ultrasonographic criteria. Preliminary results. Med Ultrason 16 285-290, 2014

4. Argenziano G, Zalaudek I, Ferrara G, Hofmann-Wellenhof R and Soyer HP: Proposal of a new classification system for melanocytic naevi. Br J Dermatol 157: 217-227, 2007.
5. Tsao H, Bevona C, Goggins $\mathrm{W}$ and Quinn T: The transformation rate of moles (melanocytic nevi) into cutaneous melanoma: A population-based estimate. Arch Dermatol 139: 282-288, 2003.

6. Goldstein AM and Tucker MA: Dysplastic Nevi and Melanoma. Cancer Epidemiol Biomarkers Prev 22: 528-532, 2013.

7. Breitbart EW, Waldmann A, Nolte S, Capellaro M, Greinert R, Volkmer B and Katalinic A: Systematic skin cancer screening in Northern Germany. J Am Acad Dermatol 66: 201-211, 2012.

8. Bafounta ML, Beauchet A, Aegerter P and Saiag P: Is dermoscopy (epiluminescence microscopy) useful for the diagnosis of melanoma? Results of a meta-analysis using techniques adapted to the evaluation of diagnostic tests. Arch Dermatol 137: 1343-1350, 2001.

9. Stolz W, Schiffner R, Pillet L, Vogt T, Harms H, Schindewolf T, Landthaler M and Abmayr W: Improvement of monitoring of melanocytic skin lesions with the use of a computerized acquisition and surveillance unit with a skin surface microscopic television camera. J Am Acad Dermatol 35: 202-207, 1996.

10. Braun RP, Lemonnier E, Guillod J, Skaria A, Salomon D, Saurat JH: Two types of pattern modification detected on the follow-up of benign melanocytic skin lesions by digitized epiluminescence microscopy. Melanoma Res 8: 431-437, 1998.

11. Haenssle HA, Krueger U, Vente C, Thoms KM, Bertsch HP, Zutt M, Rosenberger A, Neumann C and Emmert S: Results from an observational trial: Digital epiluminescence microscopy follow-up of atypical nevi increases the sensitivity and the chance of success of conventional dermoscopy in detecting melanoma. J Invest Dermatol 126: 980-985, 2006.

12. Argenziano G, Mordente I, Ferrara G, Sgambato A, Annese P and Zalaudek I: Dermoscopic monitoring of melanocytic skin lesions: Clinical outcome and patient compliance vary according to follow-up protocols. Br J Dermatol 159: 331-336, 2008.

13. Robinson JK and Nickoloff BJ: Digital epiluminescence microscopy monitoring of high-risk patients. Arch Dermatol 140: 49-56, 2004.

14. Kardynal A and Olszewska M: Modern non-invasive diagnostic techniques in the detection of early cutaneous melanoma. J Dermatol Case Rep 8: 1-8, 2014.

15. Bauer J, Blum A, Strohhäcker U and Garbe C: Surveillance of patients at high risk for cutaneous malignant melanoma using digital dermoscopy. Br J Dermatol 152: 87-92, 2005.

16. Haenssle HA, Vente C, Bertsch HP, Rupprecht R, Abuzahra F, Junghans V, Ellinghaus B, Emmert S, Hallermann C, Rosenberger A, et al: Results of a surveillance programme for patients at high risk of malignant melanoma using digital and conventional dermoscopy. Eur J Cancer Prev 13: 133-138, 2004.

17. Kittler H, Pehamberger H, Wolff K and Binder M: Follow-up of melanocytic skin lesions with digital epiluminescence microscopy: Patterns of modifications observed in early melanoma, atypical nevi, and common nevi. J Am Acad Dermatol 43: 467-476, 2000.

18. Gandini S, Sera F, Cattaruzza MS, Pasquini P, Zanetti R, Masini C, Boyle P and Melchi CF: Meta-analysis of risk factors for cutaneous melanoma: III. Family history, actinic damage and phenotypic factors. Eur J Cancer 41: 2040-2059, 2005.

19. Haenssle HA, Korpas B, Hansen-Hagge C, Buhl T, Kaune KM, Johnsen S, Rosenberger A, Schön MP and Emmert S: Selection of patients for long-term surveillance with digital dermoscopy by assessment of melanoma risk factors. Arch Dermatol 146: 257-264, 2010.

20. Schiffner R, Schiffner-Rohe J, Landthaler M and Stolz W: Long-term dermoscopic follow-up of melanocytic naevi: Clinical outcome and patient compliance. Br J Dermatol 149: 79-86, 2003.

21. Papageorgiou V, Apalla Z, Sotiriou E, Papageorgiou C, Lazaridou E, Vakirlis S, Ioannides D and Lallas A: The limitations of dermoscopy: False-positive and false-negative tumours. J Eur Acad Dermatol Venereol 32: 879-888, 2018.

22. Dasgeb B, Morris MA, Mehregan D and Siegel EL: Quantified ultrasound elastography in the assessment of cutaneous carcinoma. Br J Radiol 88: 20150344, 2015.

23. Lallas A, Apalla Z, Argenziano G, Longo C, Moscarella E, Specchio F, Raucci $M$ and Zalaudek I: The dermatoscopic universe of basal cell carcinoma. Dermatol Pract Concept 4: 11-24, 2014.

24. de Giorgi V, Gori A, Savarese I, D'Errico A, Grazzini M, Papi F, Maio V, Covarelli P, Urso C and Massi D: Teledermoscopy in doubtful melanocytic lesions: Is it really useful? Int J Dermatol 55: 1119-1123, 2016.

25. Lupu M, Caruntu C, Popa MI, Voiculescu VM, Zurac S and Boda D: Vascular patterns in basal cell carcinoma: Dermoscopic, confocal and histopathological perspectives. Oncol Lett 17: 4112-4125, 2019. 
26. Bakos RM, Bakos L, Cartell A, Manzoni AP and Prati C: Radial streaking: Unusual dermoscopic pattern in pigmented superficial basal cell carcinoma. J Eur Acad Dermatol Venereol 21: 1263-1265, 2007.

27. Lallas A, Argenziano G, Zendri E, Moscarella E, Longo C, Grenzi L, Pellacani G and Zalaudek I: Update on non-melanoma skin cancer and the value of dermoscopy in its diagnosis and treatment monitoring. Expert Rev Anticancer Ther 13: 541-558, 2013.

28. Altamura D, Menzies SW, Argenziano G, Zalaudek I, Soyer HP, Sera F, Avramidis M, DeAmbrosis K, Fargnoli MC and Peris K Dermatoscopy of basal cell carcinoma: Morphologic variability of global and local features and accuracy of diagnosis. J Am Acad Dermatol 62: 67-75, 2010.

29. Schulze HJ, Cribier B, Requena L, Reifenberger J, Ferrándiz C, Garcia Diez A, Tebbs V and McRae S: Imiquimod 5\% cream for the treatment of superficial basal cell carcinoma: Results from a randomized vehicle-controlled phase III study in Europe. Br J Dermatol 152: 939-947, 2005.

30. Mun JH, Jwa SW, Song M, Ko HC, Kim BS, Kim MB and Kim HS: Pitfalls of using dermatoscopy in defining surgical margins of basal cell carcinoma. Dermatol Surg 37: 1704-1705, 2011.

31. Micantonio T, Fargnoli MC, Piccolo D and Peris K: Letter: Changes in dermoscopic features in superficial basal cell carcinomas treated with imiquimod. Dermatol Surg 33 1403-1405, 2007.

32. Botar-Jid CM, Cosgarea R, Bolboacă SD, Şenilă SC, Lenghel LM, Rogojan L and Dudea SM: Assessment of cutaneous melanoma by use of very- high-frequency ultrasound and real-time elastography. AJR Am J Roentgenol 206: 699-704, 2016.

33. Mandava A, Ravuri PR and Konathan R: High-resolution ultrasound imaging of cutaneous lesions. Indian J Radiol Imaging 23: 269-277, 2013.

34. Cammarota T, Pinto F, Magliaro A and Sarno A: Current uses of diagnostic high-frequency US in dermatology. Eur J Radiol 27 (Suppl 2): S215-S223, 1998.

35. Crisan M, Crisan D, Sannino G, Lupsor M, Badea R and Amzica F: Ultrasonographic staging of cutaneous malignant tumors: An ultrasonographic depth index. Arch Dermatol Res 305: 305-313, 2013.

36. Samimi M, Perrinaud A, Naouri M, Maruani A, Perrodeau E, Vaillant L and Machet L: High-resolution ultrasonography assists the differential diagnosis of blue naevi and cutaneous metastases of melanoma. Br J Dermatol 163: 550-556, 2010

37. Harland CC, Kale SG, Jackson P, Mortimer PS and Bamber JC: Differentiation of common benign pigmented skin lesions from melanoma by high-resolution ultrasound. Br J Dermatol 143: 281-289, 2000.

38. Wortsman X: Sonography of facial cutaneous basal cell carcinoma: A first-line imaging technique. J Ultrasound Med 32: 567-572, 2013.

39. Gambichler T, Moussa G, Sand M, Sand D, Altmeyer P and Hoffmann K: Applications of optical coherence tomography in dermatology. J Dermatol Sci 40: 85-94, 2005.

40. Gambichler T, Regeniter P, Bechara FG, Orlikov A, Vasa R, Moussa G, Stücker M, Altmeyer P and Hoffmann K: Characterization of benign and malignant melanocytic skin lesions using optical coherence tomography in vivo. J Am Acad Dermatol 57: 629-637, 2007.

41. Guitera P,Li LX, Crotty K, Fitzgerald P, Mellenbergh R, Pellacani G and Menzies SW: Melanoma histological Breslow thicknes predicted by $75-\mathrm{MHz}$ ultrasonography. Br J Dermatol 159: 364-369, 2008

42. Nassiri-Kashani M, Sadr B, Fanian F, Kamyab K, Noormohammadpour P, Shahshahani MM, Zartab H, Naghizadeh MM, Sarraf-Yazdy M and Firooz A: Pre-operative assessment of basal cell carcinoma dimensions using high frequency ultrasonography and its correlation with histopathology. Skin Res Technol 19: e132-e138, 2013.

43. Manea A, Crisan D, Badea AF, Dumitrascu ID, Baciut MF Bran S, Mitre I, Crisan M and Baciut G: The value of ultrasound diagnosis in the multidisciplinary approach of cutaneous tumours. Case report. Med Ultrason 1: 108-110, 2018.

44. Catalano O, Caracò C, Mozzillo N and Siani A: Locoregional spread of cutaneous melanoma: Sonography findings. AJR Am J Roentgenol 194: 735-745, 2010

45. Schmid-Wendtner MH and Dill-Müller D: Ultrasound technology in dermatology. Semin Cutan Med Surg 27: 44-51, 2008.

46. Wortsman $X$ and Wortsman J: Clinical usefulness of variable-frequency ultrasound in localized lesions of the skin. J Am Acad Dermatol 62: 247-256, 2010.
47. Harland CC, Bamber JC, Gusterson BA and Mortimer PS: High frequency, high resolution B-scan ultrasound in the assessment of skin tumours. Br J Dermatol 128: 525-532, 1993.

48. Bobadilla F, Wortsman X, Muñoz C, Segovia L, Espinoza M and Jemec GB: Pre-surgical high resolution ultrasound of facial basal cell carcinoma: Correlation with histology. Cancer Imaging 8: 163-172, 2008

49. Pellacani G and Seidenari S: Preoperative melanoma thickness determination by $20-\mathrm{MHz}$ sonography and digital videomicroscopy in combination. Arch Dermatol 139: 293-298, 2003.

50. Jambusaria-Pahlajani A, Schmults CD, Miller CJ,Shin D, Williams J, Kurd SK and Gelfand JM: Test characteristics of high-resolution ultrasound in the preoperative assessment of margins of basal cell and squamous cell carcinoma in patients undergoing Mohs micrographic surgery. Dermatol Surg 35: 9-16, 2009.

51. Ianosi SL, Forsea AM, Lupu M, Ilie MA, Zurac S, Boda D, Ianosi G, Neagoe D, Tutunaru C, Popa CM, et al: Role of modern imaging techniques for the in vivo diagnosis of lichen planus. Exp Ther Med 17: 1052-1060, 2019.

52. Cioplea M, Caruntu C,Zurac S, Bastian A, Sticlaru L, Cioroianu A, Boda D, Jugulete G, Nichita L and Popp C: Dendritic cell distribution in mycosis fungoides vs. inflammatory dermatosis and other T-cell skin lymphoma. Oncol Lett 17: 4055-4059, 2019.

53. Rao BK: Atlas of Confocal Microscopy in Dermatology. 1st edition. NIDIskin LLC, New York, NY, p166, 2013.

54. Hofmann-Wellenhoff R, Pellacani G, Malvehy J and Soyer HP (eds): Reflectance Confocal Microscopy for Skin Diseases. Springer-Verlag, Berlin, 2012.

55. Pellacani G, Guitera P, Longo C, Avramidis M, Seidenari S and Menzies S: The impact of in vivo reflectance confocal microscopy for the diagnostic accuracy of melanoma and equivocal melanocytic lesions. J Invest Dermatol 127: 2759-2765, 2007.

56. Diaconeasa A, Boda D, Solovan C, Enescu DM, Vîlcea AM and Zurac S: Histopathologic features of Spitzoid lesions in different age groups. Rom J Morphol Embryol 54: 51-62, 2013.

57. Căruntu C, Boda D, Gutu DE and Căruntu A: In vivo reflectance confocal microscopy of basal cell carcinoma with cystic degeneration. Rom J Morphol Embryol 55: 1437-1441, 2014.

58. Ghita MA, Caruntu C, Rosca AE, Kaleshi H, Caruntu A, Moraru L, Docea AO, Zurac S, Boda D, Neagu M, et al: Reflectance confocal microscopy and dermoscopy for in vivo, non-invasive skin imaging of superficial basal cell carcinoma. Oncol Lett 11: 3019-3024, 2016.

59. Rishpon A, Kim N, Scope A, Porges L, Oliviero MC, Braun RP, Marghoob AA, Fox CA and Rabinovitz HS: Reflectance confocal microscopy criteria for squamous cell carcinomas and actinic keratoses. Arch Dermatol 145: 766-772, 2009.

60. LupuM,Caruntu A,CaruntuC,BodaD,MoraruL,VoiculescuVand Bastian A: Non-invasive imaging of actinic cheilitis and squamous cell carcinoma of the lip. Mol Clin Oncol 8: 640-646, 2018.

61. Ianosi SL, Batani A, Ilie MA, Tampa M, Georgescu SR, Zurac S, Boda D, Ianosi NG, Neagoe D, Calina D, et al: Non-invasive imaging techniques for the in vivo diagnosis of Bowen's disease: Three case reports. Oncol Lett 17: 4094-4101, 2019.

62. Seidenari S, Arginelli F, Bassoli S, Cautela J, French PM, Guanti M, Guardoli D, Konig K, Talbot C and Dunsby C: Multiphoton laser microscopy and fluorescence lifetime imaging for the evaluation of the skin. Dermatol Res Pract 2012: 810749, 2012.

63. Lin SJ, Jee SH, Kuo CJ, Wu RJ Jr, Lin WC, Chen JS, Liao YH, Hsu CJ, Tsai TF, Chen YF, et al: Discrimination of basal cell carcinoma from normal dermal stroma by quantitative multiphoton imaging. Opt Lett 31: 2756-2758, 2006.

64. Braun RP, Mangana J, Goldinger S, French L, Dummer R and Marghoob AA: Electrical impedance spectroscopy in skin cancer diagnosis. Dermatol Clin 35: 489-493, 2017.

65. Zhao J, Zeng H, Kalia S and Lui H: Using Raman spectroscopy to detect and diagnose skin cancer in vivo. Dermatol Clin 35: 495-504, 2017.

66. Zhang J, Fan Y, Song Y and Xu J: Accuracy of Raman spectroscopy for differentiating skin cancer from normal tissue. Medicine (Baltimore) 97: e12022, 2018

67. Zhao J, Zeng H, Kalia S and Lui H: Wavenumber selection based analysis in Raman spectroscopy improves skin cancer diagnostic specificity. Analyst (Lond) 141: 1034-1043, 2016.

68. Crisan D, Gheuca Solovastru L, Crisan M and Badea R: Cutaneous histiocytoma - histological and imaging correlations. A case report. Med Ultrason 16: 268-270, 2014.

This work is licensed under a Creative Commons Attribution-NonCommercial-NoDerivatives 4.0 International (CC BY-NC-ND 4.0) License. 